\title{
New fixed point theorems of condensing mappings satisfying the interior condition in Banach spaces
}

\author{
Shujia Chen, Shaoyuan $\mathrm{Xu}^{*}$ \\ Department of Mathematics and Statistics, Hanshan Normal University, Chaozhou, China \\ * corresponding author
}

\section{Email address:}

xushaoyuan@126.com (Shaoyuan Xu),1076429058@qq.com (Shujia Chen)

\section{To cite this article:}

Shujia Chen, Shaoyuan Xu. New Fixed Point Theorems of Condensing Mappings Satisfying the Interior Condition in Banach Space. Pure and Applied Mathematics Journal. Vol. 3, No. 6, 2014, pp. 126-131. doi: 10.11648/j.pamj.20140306.13

\begin{abstract}
In this paper, based on a basic result on condensing mappings satisfying the interior condition, some new fixed point theorems of the condensing mappings of this kind are obtained. As a result, the famous Petryshyn's theorem and some results of the Reference [8] are extended to the condensing mappings satisfying the interior condition.
\end{abstract}

Keywords: Condensing Mappings, Interior Condition, Fixed Point, Banach Space

\section{Introduction and Preliminaries}

As is well known, the condensing mappings are a class of important nonlinear operators, existing extensively in the nonlinear differential and integral equations. So, the fixed point theorems of the condensing mappings play a central role in the study of existence of the solutions of these equations (see [1-8]).

Let $X$ be a real Banach space and $D$ an open subset of $X$ with $\theta \in D$ where $\theta$ denotes the zero element of $X$. Denote by $\bar{D}$ and $\partial D$ the closure and boundary of $D$ respectively. It is well known that if $D$ is bounded and if $A: \bar{D} \rightarrow X$ is a condensing mapping, then we have had some well-known theorems as follows (see [1-5, 8]).

Theorem 1.1 Suppose that one of the following conditions is satisfied:

(i) (Leray - Schauder) $A x \neq \lambda x, \forall x \in \partial D$;

(ii) (Roth) $\|A(x)\| \leq\|x\|, \forall x \in \partial \Omega$;

(iii) (Petryshyn) $\|A(x)\| \leq\|A(x)-x\|, \forall x \in \partial \Omega$;

(iv) (Altman) $\|A(x)-x\|^{2} \geq\|A(x)\|^{2}-\|x\|^{2}, \forall x \in \partial \Omega$.

Then A has at least one fixed point in $\bar{D}$.

Recently, Antonio and Morales [7] introduce a new condition which resembles the Leray-Schauder boundary condition mentioned above. It is called the Interior Condition defined as follows.
Definition $1.1^{[7]}$ We say that a mapping $A: \bar{D} \rightarrow X$ satisfies the Interior Condition if there exists $\delta>0$ such that

$$
A x \neq \lambda x, \forall x \in D^{*}, \lambda>1, A x \notin \bar{D},
$$

where

$$
D^{*}=\{x \in D: \operatorname{dist}(x, \partial x)<\delta\}
$$

Recall that the set-measure of non-compactness of a bounded subset $E$ of $X$ is defined as (see [2-4])

$\gamma[E]=\inf \{d>0: E$ can be covered by finitely many sets of diameter $\leq d\}$ :

$A$ mapping $A: D \subset X \rightarrow X$ is called a condensing mapping (see [2 - 4]), if $A$ is continuous and $\gamma[A[E]]<\gamma[E]$ for all bounded subset $E$ of $D$ where $\gamma[E]>0$.

Definition $1.2^{[7]}$ Let $X$ be a real Banach space and $D$ an bounded open subset of $X$ with $\theta \in D$. We say that $D$ is strictly star-shaped with respect to the origin if the following condition is satisfied:

$$
\{t x: t>0\} \cap \partial G=\{x\}, \forall x \in \partial G .
$$

From now on, we will assume without loss of generality, 
that the star-shaped assumption will always be considered with respect to the origin, unless the contrary is mentioned.

In this paper, based on a basic result on condensing mappings satisfying the interior condition, some new fixed point theorems of the condensing mappings of this kind are obtained. As a result, the famous Petryshyn's theorem and some results of the Reference [8] are extended to the condensing mappings satisfying the interior condition.

\section{Main Results}

Lemma $2.1^{[7]}$ Let $X$ be a real Banach space and let $D$ be a bounded open and strictly star-shaped subset of $X$ with $\theta \in D$. If $A: \bar{D} \rightarrow X$ is a condensing mapping satisfying the Condition (I-C): there exists $\delta>0$ such that

$$
A x \neq \lambda x, \forall x \in D^{*}, \lambda>1, A x \notin \bar{D},
$$

where

$$
D^{*}=\{x \in D: \operatorname{dist}(x, \partial G)<\delta\}
$$

for some $\delta>0$, then $A$ has at least one fixed point in $\bar{D}$.

We are now in the position to apply the above basic result to derive some new fixed point theorems for the condensing mappings satisfying the Condition (I-C) which extend many well-known results to the case of the mappings satisfying the interior condition.

Theorem 2.1 Let $X$ be a real Banach space and let $D$ be a bounded open and strictly star-shaped subset of $X$ with $\theta \in D$. Let $A: \bar{D} \rightarrow X$ be a condensing mapping. Suppose also that there exist $1 \leq \alpha<\beta$ or $1<\alpha \leq \beta$ such that

$$
\begin{gathered}
\|A x-x\|^{\alpha}\|x\|^{\beta} \geq\|A x\|^{\alpha}\|A x+x\|^{\beta}-\|A x\|^{\alpha}\|x\|^{\beta} \\
\forall x \in D^{*} \text { and } A x \notin \bar{D}
\end{gathered}
$$

where

$$
D^{*}=\{x \in D: \operatorname{dist}(x, \partial G)<\delta\}
$$

for some $\delta>0$, then $A$ has at least one fixed point in $\bar{D}$.

Proof If the operator A has a fixed point on $\partial D$, then $A$ has at least one fixed point on $D$. Now suppose that A has no fixed points on $\partial D$. Next we shall prove that the condition (I-C) is satisfied.

Suppose it is not true. Then there exist $x_{0} \in \partial D$ and $\mu_{0} \geq 1$ such that $A x_{0}=\mu_{0} x_{0}$. It is easy to see that $\mu_{0}>1$. Now, consider the function defined by

$$
f(t)=(t-1)^{\alpha}-t^{\alpha}(t+1)^{\beta}+t^{\alpha}, \forall t \geq 1 .
$$

Since

$$
f^{\prime}(t)=\alpha(t-1)^{\alpha-1}-\alpha t^{\alpha-1}(t+1)^{\beta}+\alpha t^{\alpha-1}-\beta t^{\alpha}(t+1)^{\beta-1}
$$

$<0$ by formal differentiation, $f(t)$ is a strictly decreasing function in $[1, \infty)$. And so $f(t)<f(1)$ for $t>1$, i.e.,

$(t-1)^{\alpha}<t^{\alpha}(t+1)^{\beta}-t^{\alpha}$, for any $t>1$. Consequently, noting that $\left\|x_{0}\right\| \neq 0, \mu_{0}>1$, we have

$$
\begin{aligned}
& \left\|A x_{0}-x_{0}\right\|^{\alpha}\left\|x_{0}\right\|^{\beta} \\
= & \left\|\mu_{0} x_{0}-x_{0}\right\|^{\alpha}\left\|x_{0}\right\|^{\beta} \\
= & \left(\mu_{0}-1\right)^{\alpha}\left\|x_{0}\right\|^{\alpha+\beta} \\
< & {\left[\mu_{0}^{\alpha}\left(\mu_{0}+1\right)^{\beta}-\mu_{0}^{\alpha}\right]\left\|x_{\alpha}\right\|^{\alpha+\beta} } \\
= & \left\|A x_{0}\right\|^{\alpha}\left\|A x_{0}+x_{0}\right\|^{\beta}-\left\|A x_{0}\right\|^{\alpha}\left\|x_{0}\right\|^{\beta},
\end{aligned}
$$

which is a contradiction to (2.1), and so the condition (I-C) is satisfied. Therefore, it follows from Lemma 2.1 that the conclusion of Theorem 2.1 holds.

From Theorem 2.1, we can easily get the subsequent four corollaries.

Corollary 2.1 Let $X$ be a real Banach space and let $D$ be a bounded open and strictly star-shaped subset of $X$ with $\theta \in D$. Let $A: \bar{D} \rightarrow X$ be a condensing mapping. Suppose also that there exists $\alpha>1$, such that

$$
\begin{gathered}
(\|A x-x\|\|x\|)^{\alpha} \geq(\|A x\|\|A x+x\|)^{\alpha}-(\|A x\|\|x\|)^{\alpha}, \\
\forall x \in D^{*} \text { and } A x \notin \bar{D}
\end{gathered}
$$

where

$$
D^{*}=\{x \in D: \operatorname{dist}(x, \partial G)<\delta\}
$$

for some $\delta>0$, then $A$ has at least one fixed point in $\bar{D}$.

Proof Take $\alpha=\beta$. It follows from Theorem 2.1 that the conclusion of Corollary 2.1 holds true.

Corollary 2.2 Let $X$ be a real Banach space and let $D$ be a bounded open and strictly star-shaped subset of $X$ with $\theta \in D$. Let $A: \bar{D} \rightarrow X$ be a condensing mapping. Suppose also that $A$ satisfies the following condition

$$
\begin{gathered}
\sqrt{(\|A x-x\|\|x\|)^{3}} \geq \sqrt{(\|A x\|\|A x+x\|)^{3}}-\sqrt{(\|A x\|\|x\|)^{3}} ; \\
\forall x \in D^{*} \text { and } A x \notin \bar{D}
\end{gathered}
$$

where

$$
D^{*}=\{x \in D: \operatorname{dist}(x, \partial G)<\delta\}
$$

for some $\delta>0$, then $A$ has at least one fixed point in $\bar{D}$. Proof Take $\alpha=\beta=\frac{3}{2}$. It follows from Theorem 2.1 that 
the conclusion of Corollary 2.2 holds true.

Corollary 2.3 Let $X$ be a real Banach space and let $D$ be a bounded open and strictly star-shaped subset of $X$ with $\theta \in D$. Let $A: \bar{D} \rightarrow X$ be a condensing mapping. Suppose also that $A$ satisfies the following condition

$$
\begin{gathered}
(\|A x-x\|\|x\|)^{2} \geq(\|A x\|\|A x+x\|)^{2}-(\|A x\|\|x\|)^{2} ; \\
\forall x \in D^{*} \text { and } A x \notin \bar{D} ;
\end{gathered}
$$

where

$$
D^{*}=\{x \in D: \operatorname{dist}(x, \partial G)<\delta\}
$$

for some $\delta>0$, then $A$ has at least one fixed point in $\bar{D}$.

Proof Take $\alpha=\beta=2$. It follows from Theorem 2.1 that the conclusion of Corollary 2.3 holds true.

Corollary 2.4 Let $X$ be a real Banach space and let $D$ be a bounded open and strictly star-shaped subset of $X$ with $\theta \in D$. Let $A: \bar{D} \rightarrow X$ be a condensing mapping. Suppose also that there exist $\beta>1$, such that

$$
\begin{gathered}
\|A x-x\|\|x\|^{\beta} \geq\|A x\|\|A x+x\|^{\beta}-\|A x\|\|x\|^{\beta}, \\
\forall x \in D^{*} \text { and } A x \notin \bar{D},
\end{gathered}
$$

where

$$
D^{*}=\{x \in D: \operatorname{dist}(x, \partial G)<\delta\}
$$

for some $\delta>0$, then $A$ has at least one fixed point in $\bar{D}$.

Proof Take $\alpha=1$. It follows from Theorem 2.1 that the conclusion of Corollary 2.4 holds true.

Theorem 2.2 Let $X$ be a real Banach space and let $D$ be a bounded open and strictly star-shaped subset of $X$ with $\theta \in D$. Let $A: \bar{D} \rightarrow X$ be a condensing mapping. Suppose also that there exist $\alpha \geq 1, \beta \geq 0$, such that

$$
\begin{gathered}
\|A x+x\|^{\alpha+\beta} \leq\|A x\|^{\beta}\|A x-x\|^{\alpha}+\|x\|^{\alpha+\beta} ; \\
\forall x \in D^{*} \text { and } A x \notin \bar{D},
\end{gathered}
$$

where

$$
D^{*}=\{x \in D: \operatorname{dist}(x, \partial G)<\delta\}
$$

for some $\delta>0$, then $A$ has at least one fixed point in $\bar{D}$.

Proof If the operator A has a fixed point on $\partial D$, then $A$ has at least one fixed point on $D$. Now suppose that A has no fixed points on $\partial D$. Next we shall prove that the condition (I-C) is satisfied.

Suppose it is not true. Then there exist $x_{0} \in \partial D$ and $\mu_{0} \geq 1$ such that $A x_{0}=\mu_{0} x_{0}$. It is easy to see that $\mu_{0}>1$. Now, consider the function defined by

$$
f(t)=(t+1)^{\alpha+\beta}-t^{\beta}(t-1)^{\alpha}-1, \forall t \geq 1 .
$$

Since

$$
f^{\prime}(t)=\alpha\left[(t+1)^{\alpha+\beta-1}-t^{\beta}(t-1)^{\alpha}\right]+\beta\left[(t+1)^{\alpha+\beta-1}-t^{\beta-1}(t-1)^{\alpha}\right]
$$

$>0$ by formal differentiation, $f(t)$ is a strictly decreasing function in $[1, \infty)$. And so $f(t)>f(1)$ for $t>1$, Thus, $(t+1)^{\alpha+\beta}>t^{\beta}(t-1)^{\alpha}+1$, for any $t>1$. Consequently, noting that $\left\|x_{0}\right\| \neq 0, \mu_{0}>1$, we have

$$
\begin{aligned}
& \left\|A x_{0}+x_{0}\right\|^{\alpha+\beta} \\
= & \left\|\mu_{0} x_{0}+x_{0}\right\|^{\alpha+\beta} \\
= & \left(\mu_{0}+1\right)^{\alpha+\beta}\left\|x_{0}\right\|^{\alpha+\beta} \\
> & {\left[\mu_{0}^{\beta}\left(\mu_{0}-1\right)^{\alpha}+1\right]\left\|x_{0}\right\|^{\alpha+\beta} } \\
= & \left\|\mu_{0} x_{0}\right\|^{\beta}\left\|\mu_{0} x_{0}-x_{0}\right\|^{\alpha}+\left\|x_{0}\right\|^{\alpha+\beta} \\
= & \left\|A x_{0}\right\|^{\beta}\left\|A x_{0}-x_{0}\right\|^{\alpha}+\left\|x_{0}\right\|^{\alpha+\beta},
\end{aligned}
$$

which is a contradiction to (2.2), and so the condition (I-C) is satisfied. Therefore, it follows from Lemma 2.1 that the conclusion of Theorem 2.2holds.

From Theorem 2.2, we can easily obtain the subsequent five corollaries.

Corollary 2.5 Let $X$ be a real Banach space and let $D$ be a bounded open and strictly star-shaped subset of $X$ with $\theta \in D$. Let $A: \bar{D} \rightarrow X$ be a condensing mapping. Suppose also that there exists $\alpha>1$ such that

$$
\begin{gathered}
\|A x+x\|^{2 \alpha} \leq\|A x\|^{\alpha}\|A x-x\|^{\alpha}+\|x\|^{2 \alpha}, \\
\forall x \in D^{*} \text { and } A x \notin \bar{D}
\end{gathered}
$$

where

$$
D^{*}=\{x \in D: \operatorname{dist}(x, \partial G)<\delta\}
$$

for some $\delta>0$, then $A$ has at least one fixed point in $\bar{D}$.

Proof Take $\beta=\alpha>1$. It follows from Theorem 2.2 that the conclusion of Corollary 2.5 holds true

Corollary 2.6 Let $X$ be a real Banach space and let $D$ be a bounded open and strictly star-shaped subset of $X$ with $\theta \in D$. Let $A: \bar{D} \rightarrow X$ be a condensing mapping. Suppose also that $A$ satisfies the following condition

$$
\|A x+x\|^{3} \leq(\|A x\|\|A x-x\|)^{\frac{3}{2}}+\|x\|^{3},
$$




$$
\forall x \in D^{*} \text { and } A x \notin \bar{D},
$$

where

$$
D^{*}=\{x \in D: \operatorname{dist}(x, \partial G)<\delta\}
$$

for some $\delta>0$, then $A$ has at least one fixed point in $\bar{D}$.

Proof Take $\alpha=\beta=3 / 2$. It follows from Theorem 2.2 that the conclusion of Corollary 2.6 holds true.

Corollary 2.7 Let $X$ be a real Banach space and let $D$ be a bounded open and strictly star-shaped subset of $X$ with $\theta \in D$. Let $A: \bar{D} \rightarrow X$ be a condensing mapping.

Suppose also that there exists $\alpha \geq 1_{\text {such that }}$

$$
\begin{gathered}
\|A x+x\|^{\alpha} \leq\|A x-x\|^{\alpha}+\|x\|^{\alpha}, \\
\forall x \in D^{*} \text { and } A x \notin \bar{D},
\end{gathered}
$$

where

$$
D^{*}=\{x \in D: \operatorname{dist}(x, \partial G)<\delta\}
$$

for some $\delta>0$, then $A$ has at least one fixed point in $\bar{D}$.

Proof Take $\beta=0$. It follows from Theorem 2.2 that the conclusion of Corollary 2.7 holds true

Corollary 2.8 Let $X$ be a real Banach space and let $D$ be a bounded open and strictly star-shaped subset of $X$ with $\theta \in D$. Let $A: \bar{D} \rightarrow X$ be a condensing mapping. Suppose also that $A$ satisfies the following condition

$$
\begin{gathered}
\|A x+x\|^{2} \leq\|A x-x\|^{2}+\|x\|^{2}, \\
\forall x \in D^{*} \text { and } A x \notin \bar{D},
\end{gathered}
$$

where

$$
D^{*}=\{x \in D: \operatorname{dist}(x, \partial G)<\delta\}
$$

for some $\delta>0$, then $A$ has at least one fixed point in $\bar{D}$.

Proof Take $\alpha=1, \beta=1$. It follows from Theorem 2.2 that the conclusion of Corollary 2.8 holds true.

Remark 1 We can see that the formula which satisfies the condition of Corollary 2.8 above, must also satisfies the famous Altman's theorem, namely,

$$
\|A x-x\|^{2} \geq\|A x\|^{2}+\|x\|^{2}, \forall x \in D^{*} \text { and } A x \notin \bar{D}
$$

Therefore, Corollary 2.8 and Theorem 2.2 are the useful supplements of the famous Altman's theorem.

Corollary 2.9 Let $X$ be a real Banach space and let $D$ be a bounded open and strictly star-shaped subset of $X$ with $\theta \in D$. Let $A: \bar{D} \rightarrow X$ be a condensing mapping. Suppose also that $A$ satisfies the following condition

$$
\|A x+x\|^{2} \leq\|A x-x\|^{\frac{3}{2}}\|A x\|^{\frac{1}{2}}+\|x\|^{2},
$$

$$
\forall x \in D^{*} \text { and } A x \notin \bar{D} \text {, }
$$

where

$$
D^{*}=\{x \in D: \operatorname{dist}(x, \partial G)<\delta\}
$$

for some $\delta>0$, then $A$ has at least one fixed point in $\bar{D}$.

Proof Take $\alpha=\frac{3}{2}, \beta=\frac{1}{2}$. It follows from Theorem 2.2 that the conclusion of Corollary 2.9 holds true.

Theorem 2.3 Let $X$ be a real Banach space and let $D$ be a bounded open and strictly star-shaped subset of $X$ with $\theta \in D$. Let $A: \bar{D} \rightarrow X$ be a condensing mapping. Suppose also that there exist $\alpha \geq 1, \beta \geq 0$, such that

$$
\begin{gathered}
\|A x\|^{\alpha}\|A x+x\|^{\beta} \leq\|A x\|^{\beta}\|A x-x\|^{\alpha}, \\
\forall x \in D^{*} \text { and } A x \notin \bar{D} ;
\end{gathered}
$$

where

$$
D^{*}=\{x \in D: \operatorname{dist}(x, \partial G)<\delta\}
$$

for some $\delta>0$, then $A$ has at least one fixed point in $\bar{D}$.

Proof If the operator A has a fixed point on $\partial D$, then $A$ has at least one fixed point on $D$. Now suppose that A has no fixed points on $\partial D$.Next we shall prove that the condition (I-C) is satisfied.

Suppose it is not true. Then there exist $x_{0} \in \partial D$ and $\mu_{0} \geq 1$ such that $A x_{0}=\mu_{0} x_{0}$. It is easy to see that $\mu_{0}>1$. Now, consider the function defined by

$$
f(t)=t^{\alpha}(t+1)^{\beta}-t^{\beta}(t-1)^{\alpha}, \forall t \geq 1 .
$$

Since

$f^{\prime}(t)=\alpha\left[t^{\alpha-1}(t+1)^{\beta}-(t-1)^{\alpha-1} t^{\beta}\right]+\beta\left[t^{\alpha}(t+1)^{\beta-1}-t^{\beta-1}(t-1)^{\alpha}\right]$ $>0$ by formal differentiation, $f(t)$ is a strictly decreasing function in $[1, \infty)$. And so $f(t)>f(1)$ for $t>1$, Thus, $t^{\alpha}(t+1)^{\beta}>t^{\beta}(t+1)^{\alpha}$, for any $t>1$. Consequently, noting that $\left\|x_{0}\right\| \neq 0, \mu_{0}>1$, we have

$$
\begin{aligned}
& \left\|A x_{0}\right\|^{\alpha}\left\|A x_{0}+x_{0}\right\|^{\beta} \\
= & \left\|\mu_{0} x_{0}\right\|^{\alpha}\left\|\mu_{0} x_{0}+x_{0}\right\|^{\beta} \\
= & \mu_{0}^{\alpha}\left(\mu_{0}+1\right)^{\beta}\left\|x_{0}\right\|^{\alpha+\beta} \\
> & \mu_{0}{ }^{\beta}\left(\mu_{0}-1\right)^{\alpha}\left\|x_{0}\right\|^{\alpha+\beta}
\end{aligned}
$$




$$
\begin{aligned}
& =\left\|u_{0} x_{0}\right\|^{\beta}\left\|\mu_{0} x_{0}-x_{0}\right\|^{\alpha} \\
& =\left\|A x_{0}\right\|^{\beta}\left\|A x_{0}-x_{0}\right\|^{\alpha},
\end{aligned}
$$

which is a contradiction to (2.3), and so the condition (I-C) is satisfied. Therefore, it follows from Lemma 2.1 that the conclusion of Theorem 2.3 holds.

From Theorem 2.3 we can easily get the subsequent such a corollary.

Corollary 2.10 Let $X$ be a real Banach space and let $D$ be a bounded open and strictly star-shaped subset of $X$ with $\theta \in D$. Let $A: \bar{D} \rightarrow X$ be a condensing mapping. Suppose also that $A$ satisfies the following condition

$$
\|A x\| \leq\|A x-x\|, \forall x \in D^{*} \text { and } A x \notin \bar{D} ;
$$

where

$$
D^{*}=\{x \in D: \operatorname{dist}(x, \partial G)<\delta\}
$$

for some $\delta>0$, then $A$ has at least one fixed point in $\bar{D}$. Proof Take $\alpha=1, \beta=0$. It follows from Theorem 2.3 that the conclusion of Corollary 2.10 holds true.

Remark 2 Corollary 2.10 is the famous Petryshyn' s theorem, so the Theorem 2.3 extends the famous Petryshyn's theorem to the case of condensing mappings satisfying the interior condition.

Theorem 2.4 Let $X$ be a real Banach space and let $D$ be a bounded open and strictly star-shaped subset of $X$ with $\theta \in D$. Let $A: \bar{D} \rightarrow X$ be a condensing mapping. Suppose also that there exist $\alpha \geq 1, \beta \geq 0$, such that

$$
\begin{gathered}
\|A x-x\|^{\alpha}\|x\|^{\alpha+\beta} \geq\|A x\|^{\alpha}\|A x+x\|^{\alpha+\beta}-\|x\|^{2 \alpha+\beta}, \\
\forall x \in D^{*} \text { and } A x \notin \bar{D}
\end{gathered}
$$

where

$$
D^{*}=\{x \in D: \operatorname{dist}(x, \partial G)<\delta\}
$$

for some $\delta>0$, then $A$ has at least one fixed point in $\bar{D}$.

Proof If the operator A has a fixed point on $\partial D$, then $A$ has at least one fixed point on $D$. Now suppose that A has no fixed points on $\partial D$. Next we shall prove that the condition (I-C) is satisfied.

Suppose it is not true. Then there exist $x_{0} \in \partial D$ and $\mu_{0} \geq 1$ such that $A x_{0}=\mu_{0} x_{0}$. It is easy to see that $\mu_{0}>1$. Now, consider the function defined by

$$
f(t)=(t-1)^{\alpha}-t^{\alpha}(t+1)^{\alpha+\beta}+1, \forall t \geq 1 .
$$

Since

$$
f^{\prime}(t)=\alpha\left[(t-1)^{\alpha-1}-t^{\alpha}(t+1)^{\alpha+\beta}-t^{\alpha}(t+1)^{\alpha+\beta-1}\right]-\beta\left[t^{\alpha}(t+1)^{\alpha+\beta-1}\right]
$$

$<0$ by formal differentiation, $f(t)$ is a strictly decreasing function in $[1, \infty)$. And so $f(t)<f(1)$ for $t>1$, Thus, $(t-1)^{\alpha}<t^{\alpha}(t+1)^{\alpha+\beta}-1$, for any $t>1$. Consequently, noting that $\left\|x_{0}\right\| \neq 0, \mu_{0}>1$, we have

$$
\begin{aligned}
& \left\|x_{0}\right\|^{\alpha+\beta}\left\|\mu_{0} x_{0}-x_{0}\right\|^{\alpha} \\
= & \left\|A x_{0}-x_{0}\right\|^{\alpha}\left\|x_{0}\right\|^{\alpha+\beta} \\
= & \left(\mu_{0}-1\right)^{\alpha}\left\|x_{0}\right\|^{2 \alpha+\beta} \\
< & {\left[\mu_{0}^{\alpha}\left(\mu_{0}+1\right)^{\alpha+\beta}-1\right]\left\|x_{0}\right\|^{2 \alpha+\beta} } \\
= & \left\|u_{0} x_{0}\right\|^{\alpha}\left\|\mu_{0} x_{0}+x_{0}\right\|^{\alpha+\beta}-\left\|x_{0}\right\|^{2 \alpha+\beta} \\
= & \left\|A x_{0}\right\|^{\alpha}\left\|A x_{0}+x_{0}\right\|^{\alpha+\beta}-\left\|x_{0}\right\|^{2 \alpha+\beta},
\end{aligned}
$$

which is a contradiction to (2.4), and so the condition (I-C) is satisfied. Therefore, it follows from Lemma 2.1 that the conclusion of Theorem 2.4 holds.

From Theorem 2.4, we can easily obtain the subsequent three corollaries.

Corollary 2.11 Let $X$ be a real Banach space and let $D$ be a bounded open and strictly star-shaped subset of $X$ with $\theta \in D$. Let $A: \bar{D} \rightarrow X$ be a condensing mapping. Suppose also that there exists $\alpha \geq 1$ such that

$$
\begin{gathered}
\|x\|^{\alpha}\|A x-x\|^{\alpha} \geq\|A x\|^{\alpha}\|A x+x\|^{\alpha}-\|x\|^{2 \alpha}, \\
\forall x \in D^{*} \text { and } A x \notin \bar{D}
\end{gathered}
$$

where

$$
D^{*}=\{x \in D: \operatorname{dist}(x, \partial G)<\delta\}
$$

for some $\delta>0$, then $A$ has at least one fixed point in $\bar{D}$.

Proof Take $\beta=0$. It follows from Theorem 2.4 that the conclusion of Corollary 2.11 holds true.

Corollary 2.12 Let $X$ be a real Banach space and let $D$ be a bounded open and strictly star-shaped subset of $X$ with $\theta \in D$. Let $A: \bar{D} \rightarrow X$ be a condensing mapping. Suppose also that $A$ satisfies

$$
\begin{gathered}
\|x\|\|A x-x\| \geq\|A x\|\|A x+x\|-\|x\|^{2}, \\
\forall x \in D^{*} \text { and } A x \notin \bar{D},
\end{gathered}
$$

where

$$
D^{*}=\{x \in D: \operatorname{dist}(x, \partial G)<\delta\}
$$

for some $\delta>0$, then $A$ has at least one fixed point in $\bar{D}$.

Proof Take $\alpha=1, \beta=0$. It follows from Theorem 2.4 
that the conclusion of Corollary 2.12 holds true.

Corollary 2.13 Let $X$ be a real Banach space and let $D$ be a bounded open and strictly star-shaped subset of $X$ with $\theta \in D$. Let $A: \bar{D} \rightarrow X$ be a condensing mapping. Suppose also that $A$ satisfies

$$
\begin{gathered}
\|A x-x\|\|x\|^{2} \geq\|A x\|\|A x+x\|^{2}-\|x\|^{3}, \\
\forall x \in D^{*} \text { and } A x \notin \bar{D},
\end{gathered}
$$

where

$$
D^{*}=\{x \in D: \operatorname{dist}(x, \partial G)<\delta\}
$$

for some $\delta>0$, then $A$ has at least one fixed point in $\bar{D}$.

Proof Take $\alpha=1, \beta=1$. It follows from Theorem 2.4 that the conclusion of Corollary 2.13 holds true.

\section{Acknowledgements}

The authors are grateful to the referees and the editors for valuable comments and suggestions, which have improved the original manuscript greatly. The research is partially supported by the foundation of the research item of Strong Department of Engineering Innovation, Research on Fixed Point Theory with Banach Algebras in Abstract Spaces and Applications, which is sponsored by the Strong School of Engineering Innovation of Hanshan Normal University, China (2013).

\section{References}

[1] W. V. Petryshyn, Structure of the fixed points sets of $k$-set-contractions, Arch. Ration. Mach. Anal., 40 (1971/71), 312-318.

[2] K. Deimling, Nonlinear functional analysis, Springer-Verlag, New York/Berlin, 1985.

[3] A. Granas and J. Dugundji, Fixed point theory, Springer-Verlag, New York/Berlin, 2003.

[4] E. Zeidler, Nonlinear functional analysis and its applications, Springer-Verlag, New York/Berlin, 1986.

[5] R. D. Nussbaum, The fixed point index and fixed point theorems for k-set-contractions, Univ. of Chicago, Ph. D. thesis, 1969.

[6] F. E. Browder, Semicontractive and semiaccretive nonlinear mappings in Banach spaces, Bull. Amer. Math. Soc., 74 (1968), 660-665.

[7] J. M. Antonio and C.H. Morales, Fixed point theorems under the interior condition, Proc. Amer. Math. Soc., 134 (2006), 501-507.

[8] Shaoyuan Xu and Chongjun Zhu, New Fixed Point Theorems of Condensing Map-pings Satisfying the Interior Condition in Banach spaces, Anal. Theory Appl. 26 (1) (2010),43-52. 